\title{
Effectiveness of Pertuzumab, Trastuzumab, and Docetaxel Combination Neoadjuvant Chemotherapy for HER2-Positive Inflammatory Breast Cancer: A Case Report
}

\author{
Yuji Yamashita ${ }^{a}$ Yuko Tanaka $^{a}$ Seishi Kono $^{a}$ Meiko Nishimura $^{b}$ Toru Mukohara ${ }^{b}$ \\ Yukiko Morinaga $^{c}$ Shigeo Hara ${ }^{c}$ Shintaro Takao ${ }^{a}$ \\ a Division of Breast and Endocrine Surgery, Department of Surgery, Kobe University Graduate School of Medicine, Kobe, Japan; \\ ${ }^{b}$ Division of Medical Oncology/Hematology, Department of Medicine, Kobe University Graduate School of Medicine, Kobe, Japan; \\ ${ }^{\mathrm{c}}$ Division of Diagnostic Pathology, Department of Pathology, Kobe University Graduate School of Medicine, Kobe, Japan
}

\section{Established Facts}

- Inflammatory breast cancer (IBC) is the most aggressive form of primary breast cancer.

- It is a variant of breast cancer that progresses rapidly and is characterized by local recurrence, metastasis, and high mortality.

\section{Novel Insights}

- Combination neoadjuvant chemotherapy with pertuzumab, trastuzumab, and docetaxel (PTD) for HER2-positive IBC was effective, and this regimen may contribute to further improvements in the cure rate for this malignancy.

\section{Keywords}

Inflammatory breast cancer · Combination neoadjuvant chemotherapy · Multidisciplinary treatment

\section{Summary}

Background: Inflammatory breast cancer (IBC) is the most aggressive form of primary breast cancer. Case Report: A 40-year-old woman was referred to our hospital for evaluation of an induration in the right breast, suspected to be breast cancer. The tumor was diagnosed as estrogen receptor-negative, progesterone receptor-negative, HER2-positive, T4dN3cM0 stage IIIc IBC with axillary lymph node metastasis. Rather than surgical intervention, we chose a systemic treatment approach with pertuzumab, trastuzumab, and docetaxel (PTD) combination therapy which was shown to be effective for HER2-positive IBC in the NeoSphere trial. After 4 cycles of treatment, the patient had a partial response, allowing mastectomy of the right breast and axillary lymph node dissection to achieve local control. We review this case because of the success of PTD combination neoadjuvant chemotherapy for HER2-positive IBC. Conclusion: To improve the poor prognosis of IBC, combined modality therapy is required, including chemotherapy and local treatment such as surgery and/or radiation therapy. In this case, combination neoadjuvant chemotherapy with PTD for HER2-positive IBC was effective, and this regimen may contribute to further improvements in the cure rate for this malignancy.

(c) 2017 S. Karger GmbH, Freiburg

\section{KARGER}

() 2017 S. Karger GmbH, Freiburg
Dr. Yuji Yamashita

Division of Breast and Endocrine Surgery, Department of Surgery Kobe University Graduate School of Medicine

7-5-1 Kusunoki-Cho, Chuo-ku, Kobe, 650-0017, Japan yuchanyuchan1104@msn.com 


\section{Introduction}

Inflammatory breast cancer (IBC) is the most aggressive form of primary breast cancer. IBC is a clinical diagnosis, with tumor cells entering dermal lymphatic channels and causing inflammatory signs [1-3]. The incidence of IBC is $1-6 \%$ in the USA, but it varies significantly between different countries $[1,4,5]$. IBC progresses rapidly and is characterized by local recurrence, metastasis, and high mortality [6]. The average survival of 3,648 IBC patients in the Surveillance, Epidemiology, and End Results (SEER) registry was 2.9 years, which is significantly shorter than that of patients with locally advanced breast cancer $[7,8]$. Combined modality therapy with local treatment such as surgery or radiation therapy plus chemotherapy can improve the prognosis. Although guidelines for treating breast cancer subtypes are becoming available, many patients with breast cancers such as IBC that have biological characteristics with a poor prognosis, or locally advanced breast cancer, are best treated individually. Our patient with HER2-positive IBC achieved a partial response to combination neoadjuvant chemotherapy consisting of 4 cycles of pertuzumab, trastuzumab, and docetaxel (PTD), which allowed mastectomy and axillary lymph node dissection for the purpose of local control.
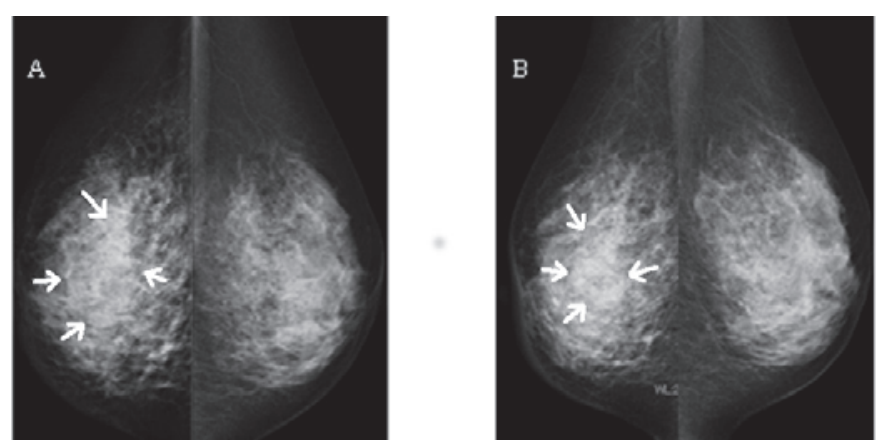

Fig. 1. Mammography of the left and right breast. A Pretreatment mammography revealed distortion in the right breast $(\rightarrow)$; B Posttreatment mammography showing improvement of the distortion $(\rightarrow)$.

\section{Case Report}

A 40-year-old woman was referred by her local hospital for an induration in the right breast suspected to be breast cancer. She presented with extensive redness and edema of the right breast skin and swelling of the axillary lymph nodes. Mammography showed distortion in the right breast (fig. $1 \mathrm{~A}$ ). Contrast-enhanced breast magnetic resonance imaging (MRI) revealed increased thickness and contrast enhancement of the skin, intraductal progression, the number of nodules, and extensive contrast enhancements in the upper region of the right breast (fig. 2 A,B). On 18F-fluoro-2-deoxy-D-glucose positron emission tomography/computed tomography (FDG-PET/CT), uptake of FDG was seen in the apocrine gland of the right breast and in the right supraclavicular, infraclavicular, and axillary lymph nodes. In other organs, no uptake of FDG was seen. IBC with axillary lymph node and skin metastases was suspected. Pathological findings following a core needle biopsy of the breast nodule indicated invasive ductal carcinoma with potential invasion of the adjacent adipose tissue and vascular system. And fine needle aspiration cytology of the axillary lymph nodes indicated adenocarcinoma. Regarding the supraclavicular and infraclavicular lymph nodes, uptake of FDG was strong (maximum standardized uptake value 2.8 and 3 , respectively), and we assumed that these nodes were tumor-infiltrated. We diagnosed the tumor as estrogen receptor-negative, progesterone receptor-negative, HER2-positive, and clinical T4dN3cM0 stage IIIc IBC with axillary lymph node metastasis. Given the diagnosis of IBC and suspected supraclavicular lymph node metastasis, systemic therapy was chosen rather than surgical intervention. Because the patient's overall condition was good with a performance status of 0 , she was treated with systemic chemotherapy. We chose not to use an anthracycline-based regimen because of a previous history of malignant lymphoma for which an anthracycline-based therapy may or may not have been used. We chose PTD, which was shown to be effective for HER2-positive IBC in the NeoSphere trial [9]. The patient received an initial dose of pertuzumab $840 \mathrm{mg}$, followed by $420 \mathrm{mg}$ every 3 weeks. Trastuzumab was administered at an initial dose of $8 \mathrm{mg} / \mathrm{kg}$, followed by $6 \mathrm{mg} / \mathrm{kg}$ every 3 weeks. Docetaxel was administered at a dose of $75 \mathrm{mg} / \mathrm{m}^{2}$ followed by the same dosage every 3 weeks. After 4 cycles of treatment, the redness and swelling of the skin improved and the induration was no longer palpable. Mammography showed improvement of the distortion (fig. $1 \mathrm{~B}$ ), and no breast nodules were seen on MRI (fig. 2 C, D). FDG/PET-CT showed uptake of FDG in the axilla, but not in the breast, the supraclavicular and infraclavicular lymph nodes, and other organs. Accordingly, we assessed that it was now possible to perform a mastectomy and axillary lymph node dissection of the right breast for the purpose of local control. In the postoperative pathology evaluation, no nodules were observed in the cut surface, and viable tumor cells were scattered throughout the lymph vessels and venous vessels. The therapeutic response was diag-
Fig. 2. Axial contrast-enhanced magnetic resonance imaging (MRI) pre- and post-chemotherapy (A, C); maximum intensity projection images of contrast-enhanced MRI pre- and post-chemotherapy (B, D). A, B MRI revealed increased thickness and contrast enhancement of the skin, intraductal progression, the number of nodules, and extensive contrast enhancements in the upper side of the right breast; C, D MRI showing absence of nodules in the breast.
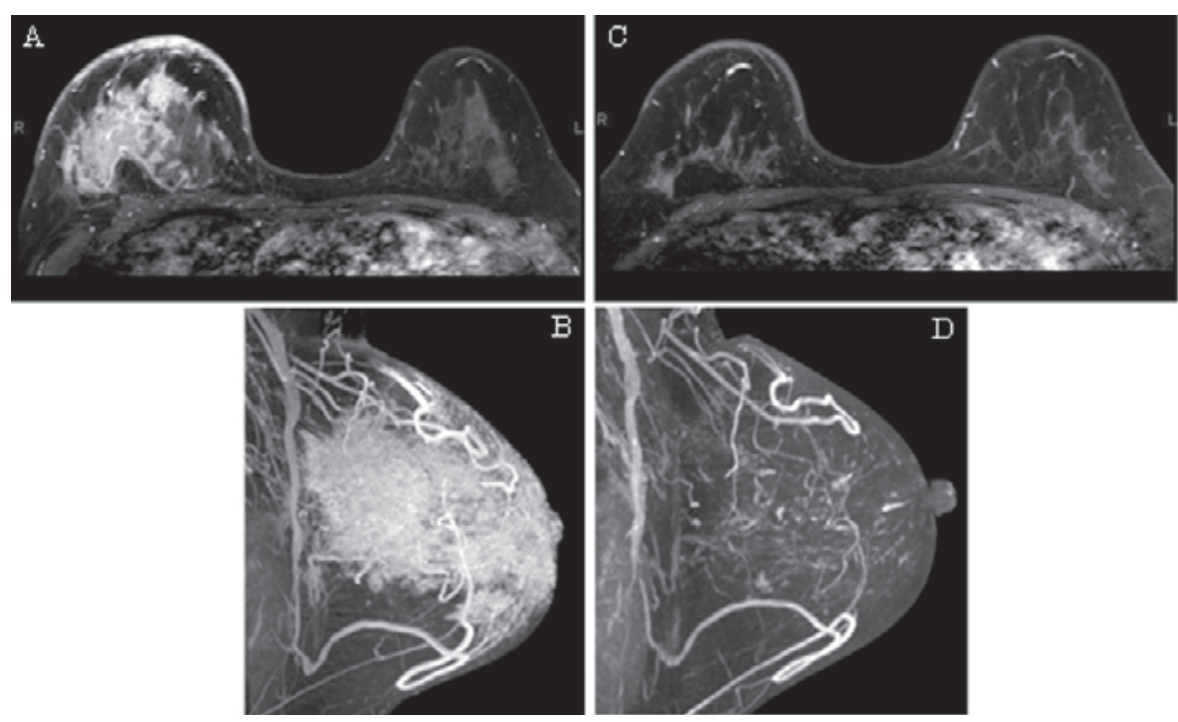
nosed as grade $2 \mathrm{~b}$, and 14 of 20 lymph nodes were involved. Although the lesion could be surgically removed for the purpose of local control, the IBC had invaded the supraclavicular and infraclavicular lymph nodes prior to chemotherapy, and the postsurgical evaluation showed a lot of remaining involved lymph nodes. Therefore, it was considered necessary to continue systemic treatment. The patient was given an additional 4 cycles of PTD followed by radiation therapy of the chest wall and supraclavicular/high fossa. After that, no new recurrence was found by diagnostic imaging, and maintenance therapy with pertuzumab and trastuzumab was given for another 6 months. At the latest followup, no recurrence was noted.

\section{Discussion}

Guidelines for the treatment of breast cancer by subtype have been recently established; however, it is often necessary to treat breast cancer with a poor prognosis, such as IBC or locally advanced breast cancer, on an individual basis. To improve prognosis, multimodal treatment of IBC, even at an early stage, is recommended because of an expected poor response to only surgery and radiation $[10,11]$. In a retrospective study, Ueno et al. [12] reported a good long-term prognosis, with 5- and 10-year overall survival (OS) rates of 40 and 33\%, respectively. Similarly, Baldini et al. [5] also reported 5- and 10-year OS rates of 44 and 32\%, respectively, with combined radiation, chemotherapy, and surgery. However, these studies did not report outcomes in HER2-positive IBC. The efficiency and safety of trastuzumab have been reported in HER2-positive breast cancer $[9,13,14]$, suggesting that its administration before or after surgery is warranted in such patients. Trastuzumab and pertuzumab were administered in combination with neoadjuvant chemotherapy for HER2-positive locally advanced breast cancer or IBC in the NeoSphere [9] and TRYPHAENA trials
[15]. The NeoSphere trial reported a pathological complete response (pCR) rate of $45.8 \%$, and in cases of hormone receptor-negative and HER2-positive breast cancer, the pCR rate was 54.4\% [9], whereas the TRYPHAENA trial reported a PCR rate of 50.7\% [15]. In both trials, the combination treatment was shown to be safe. In cases of IBC with axillary lymph node metastasis, Hennessy et al. [1] reported a good prognosis, with a 5-year OS rate of $82.5 \%$ if the patients achieved a pCR of the axillary lymph node metastasis after primary chemotherapy. Therefore, $\mathrm{pCR}$ can be considered to indicate a good prognosis during neoadjuvant chemotherapy.

In our patient, we chose PTD therapy, which does not include anthracyclines, for the systemic therapy, because anthracyclines may have been previously used to treat malignant lymphoma. PTD therapy was effective; consequently, we were able to perform surgery for local control. If the patient had been treated with PTD therapy for more than 4 courses before surgery, a further decrease in the number of involved lymph nodes or PCR might have been achieved.

Our experience with HER2-positive locally progressive IBC in this patient suggests the effectiveness of neoadjuvant combination chemotherapy with PTD for future cases. A regimen of trastuzumab plus pertuzumab with an anthracycline may become more widely used in the future [15]. In addition, neoadjuvant combination treatment comprising trastuzumab, emtansine, and pertuzumab for HER2-positive breast cancer is being evaluated [16].

\section{Disclosure Statement}

There are no conflicts of interest for all authors.

\section{References}

1 Hennessy BT, Gonzalez-Angulo AM, Hortobagyi GN, et al.: Disease-free and overall survival after pathologic complete disease remission of cytologically proven inflammatory breast carcinoma axillary lymph node metastases after primary systemic chemotherapy. Cancer 2006;106:1000-1006.

2 Tabbane F, Bahi J, Rahal K, et al.: Inflammatory symptoms in breast cancer. Correlations with growth rate, clinicopathologic variables, and evolution. Cancer 1989;64:2081-2089.

3 Jaiyesimi IA, Buzdar AU, Hortobagyi G: Inflammatory breast cancer: a review. J Clin Oncol 1992;10:10141024.

4 Levine PH, Steinhorn SC, Ries LG, Aron JL: Inflammatory breast cancer: the experience of the surveillance, epidemiology, and end results (SEER) program. J Natl Cancer Inst 1985;74:291-297.

5 Baldini E, Gardin G, Evagelista G, et al.: Long-term results of combined-modality therapy for inflammatory breast carcinoma. Clin Breast Cancer 2004;5:358-363.

6 Panades M, Olivotto IA, Speers CH, et al.: Evolving treatment strategies for inflammatory breast cancer: a population-based survival analysis. J Clin Oncol 2005; 23:1941-1950.
7 Kashiwagi S1, Ishihara S, Ishii M, et al.: Case of a patient with inflammatory breast cancer who responded to preoperative chemotherapy with paclitaxel plus bevacizumab and could subsequently undergo surgery (article in Japanese). Gan To Kagaku Ryoho 2013;40: 2384-2386.

8 Hance KW, Anderson WF, Devesa SS, et al.: Trends in inflammatory breast carcinoma incidence and survival: the surveillance, epidemiology, and end results program at the National Cancer Institute. J Natl Cancer Inst 2005;97:966-975.

9 Gianni L, Pienkowski T, Im YH, et al.: Efficacy and safety of neoadjuvant pertuzumab and trastuzumab in women with locally advanced, inflammatory, or early HER2-positive breast cancer (NeoSphere): a randomised multicentre, open-label, phase 2 trial. Lancet Oncol 2012;13:25-32.

10 Perez CA, Fields JN, Fracasso PM, et al.: Management of locally advanced carcinoma of the breast. II. Inflammatory carcinoma. Cancer 1994;74:466-476.

11 Dawood S, Ueno NT, Cristofanilli M: The medical treatment of inflammatory breast cancer. Semin Oncol 2008;35:64-71.
12 Ueno NT, Buzdar AU, Singletary SE, et al.: Combinedmodality treatment of inflammatory breast carcinoma: twenty years of experience at M. D. Anderson Cancer Center. Cancer Chemother Pharmacol 1997;40:321-329.

13 Buzdar AU, Ibrahim NK, Francis D, et al.: Significantly higher pathologic complete remission rate after neoadjuvant therapy with trastuzumab, paclitaxel, and epirubicin chemotherapy: results of a randomized trial in human epidermal growth factor receptor 2-positive operable breast cancer. J Clin Oncol 2005;23:3676-3685.

14 Untch M, Rezai M, Loibl S, et al.: Neoadjuvant treatment with trastuzumab in HER2-positive breast cancer: results from the GeparQuattro study. J Clin Oncol 2010;28:2024-2031.

15 Schneeweiss A, Chia S, Hickish T, et al.: Pertuzumab plus trastuzumab in combination with standard neoadjuvant anthracycline-containing and anthracyclinefree chemotherapy regimens in patients with HER2positive early breast cancer: a randomized phase II cardiac safety study (TRYPHAENA). Ann Oncol 2013; 24:2278-2284.

16 clinicaltrials.gov/ct2/show/study/NCT02131064. 